\title{
BRED VECTOR APPLIED TO THE ATMOSPHERIC DYNAMICS
}

Conference Paper · January 2015

DOI: 10.20906/CPS/NSC2016-0088

\section{5 authors, including:}

Luis Fernando Salgueiro Romero

Universidad Nacional de Asunción

3 PUBLICATIONS $\mathbf{0}$ CITATIONS

SEE PROFILE

Saulo R. Freitas

USRA/GESTAR - NASA Goddard Space Flight Center

305 PUBLICATIONS $\quad \mathbf{3 , 6 7 6}$ CITATIONS

SEE PROFILE

Haroldo Fraga de Campos Velho

National Institute for Space Research, Brazil

406 PUBLICATIONS 1,903 CITATIONS

SEE PROFILE

Some of the authors of this publication are also working on these related projects:

Framework for Mapping and Performance Tuning of a Weather Prediction Application on High Performance Heterogeneous Computing Systems View project

Data assimilation: using neural network for emulating NCEP-NOAA analysis (3D-Var method) View project 


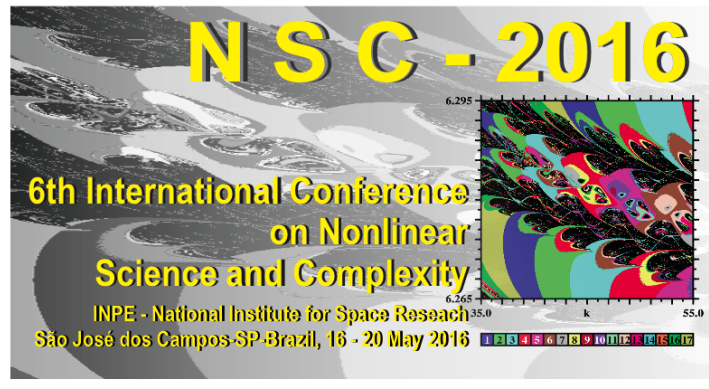

INPE - National Institute for Space Research

São José dos Campos - SP - Brazil - May 16-20, 2016

BRED VECTOR APPLIED TO THE ATMOSPHERIC DYNAMICS

\author{
Luis F. Salgueiro Romero, ${ }^{1}$, Sandra A. Sandri, ${ }^{2}$, Haroldo F. de Campos Velho, ${ }^{3}$, Rosângela S. C. \\ Cintra, ${ }^{4}$, Saulo R. Freitas ${ }^{5}$
}

\begin{abstract}
${ }^{1}$ National Institute for Space Research (INPE), São José dos Campos (SP), Brazil, luis.romero@inpe.br
${ }^{2}$ National Institute for Space Research (INPE), São José dos Campos (SP), Brazil, sandra.sandri@inpe.br

${ }^{3}$ National Institute for Space Research (INPE), São José dos Campos (SP), Brazil, haroldo@lac.inpe.br

${ }^{4}$ National Institute for Space Research (INPE), São José dos Campos (SP), Brazil, rosangela.cintra@lac.inpe.br

${ }^{5}$ National Institute for Space Research (INPE), São José dos Campos (SP), Brazil, saulo.freitas@cptec.inpe.br
\end{abstract}

\begin{abstract}
One remarkable conquer of science was the numerical weather prediction. However, some dynamics processes are not well represented in the simulation, generating a degradation of the forecasting. In addition, a chaotic atmospheric dynamics is also noted, with strong dependency of the initial condition. The initial condition is never known in a complete way. Indeed, the procedure to determine the initial condition in the operational prediction centers is called data assimilation, where the background fields (fore-casted atmospheric state) are combined with observations for producing the analysis the new initial condition. The bred vector approach can be employed to investigate the sensitivity of a certain process in the atmospheric dynamics simulation, detecting the fastest-growing instabilities. The latter scheme is also applied to evaluate the goodness of the predicted dynamics, i.e., the breeding process is employed to evaluate the predictability. The bred vector is computed as the difference between reference and perturbed simulations for the dynamical system after a time period of integration. After the mentioned time integration period, the bred vector is scaled at the same size as the initial perturbation, and a new perturbed initial condition is added to restart the process. Several simulations using the SPEEDY model was done showing regions of instabilities for some prognostics variables.
\end{abstract}

keywords: Climate Dynamics, Numerical Simulation, Atmospheric dynamics, global circulation model, bred vector.

\section{NUMERICAL WEATHER PREDICTION}

The science of weather forecasting is a complex problem embracing different areas of study such us geophysics, thermodynamics, biological interactions, mathematics and others. The direct impact that mankind activities has on these interdependent phenomenons are increasing with the past of the years making changes in the climate equilibrium. The climate change can address most frequent and intense severe weather. The countries are studying several policies for adaptation and mitigation for these negative impacts. Under this scenario, the relevance of a good weather forecasting is increased.

The weather prediction process deals with a system of initial value problem of nonlinear differential equations. These mathematical modeling tries to capture the complex nature of the atmospheric dynamics. However, the model is not able to have a exact description of the dynamics. There are many challenges to be overcame: chaotic dynamics (strongly dependent from the initial condition), turbulence parameterization, formation and/or dynamics of clouds, surface representations, multiscale phenomena, just to cite few of them.

Therefore, a previous evaluation of the goodness of the forecasting is searched. The forecasting evaluation can be quantified - predictability - by statistical methods, such as ensemble prediction, or Bred Vectors. The latter approach is suitable to quantify the quality of the forecasting, and it can be applied for further analysis such as the detection of instability regions and the influence of a certain meteorological variable in the dynamics.

\section{BRED VECTORS}

Breeding method has been used since 1992 to generate perturbation for ensemble forecasting at the NCEP, where the method simulates the development of growing errors in the analysis cycle [4]. This method, developed by Toth and Kalnay initially in the context of 
atmospheric data assimilation, make possible to find those perturbations that grows naturally in the system [5].

The Bred Vectors are computed as follows - see fig 1 , there are reference $F(\vec{x} ; t)$ and perturbed $\delta F(\vec{x} ; t)$ models, where the initial state of the second model is slightly different. The vector $\vec{x}$ represent the vector state of the model, i.e., sea surface temperature (SST), surface pressure, wind fields, temperature, density, and moisture.

Both models are integrated forward in a time interval $\Delta \mathrm{T}$. The difference between these two nonlinear dynamics are called Bred Vectors. At the time step $\Delta \mathrm{T}$, known as "breeding interval", the bred vector are scaled down to the same size as the initial perturbation, and added to the control (reference) unperturbed model, generating a new perturbed mode. The time integration process is restarted and, after the same interval $\Delta \mathrm{T}$, a second bred vector is computed.

The process is repeated in cycles until the difference between the bred vectors. It is possible to verify some saturation, where the bred vector is approximately constant. At this point, the bred vectors created are approximations of the Leading Lyapunov Vectors (L.L.V.) and like them are independent of the size (norm) chosen initially to generate the perturbation [5].

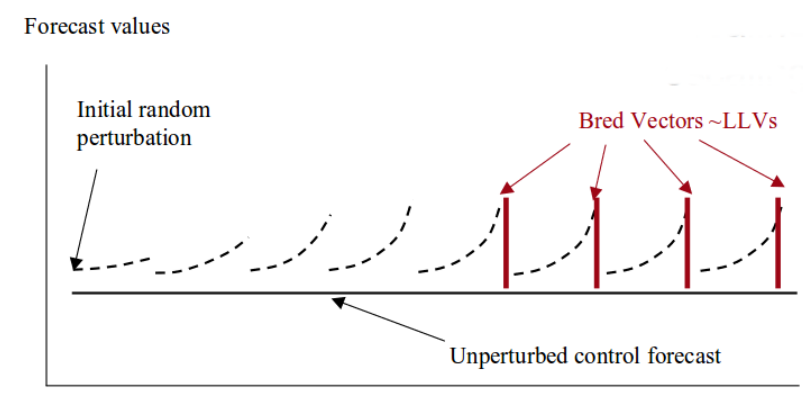

time

Figure 1 - Temporal Series of the Bred Vector in action. Source: [3]

The breeding method can insulate and to identify aspects of time-depend of nonlinear system flows. Such features are unstable to small perturbations. In addition, the bred vector are linked with high and low predictability - see [6].

\section{THE SPEEDY MODEL}

The SPEEDY (Simplified Parametrization, privitivE-Equation DYnamics) code [1], is an Atmospheric Global Circulation Model (AGCM) developed by the International Centre for Theoretical Physics (ICTP), Triesty (Italy), based on a spectral dynamical core, with a simplified set of physical parametrization schemes. The model is used for the dynamics study of the atmosphere at global scale close to numerical weather models employed in the prediction Centers [2].

The configuration of the model was T30L7, corresponding to a horizontal resolution with triangular spectral truncation at total wave-number 30 (T30), and standard Gaussian grid of 96 (longitude) by 48 (latitude) points, with seven vertical vertical pressure levels (100, 200, 300, 500, 700, 850, $925 \mathrm{HPa}$ ) [1, 2].

The prognostic variables of input and output model are the absolute temperature $(T)$, surface pressure $(p s)$, zonal wind $(u)$, southern wind $(v)$, and specific humidity (q) $[2]$.

\section{RESULTS AND DISCUSSION}

The period of simulation was from 01/Jan/1982 00 UTC up to $07 / \mathrm{Jan} / 198218$ UTC, where the data assimilation has a cycle of 6 Hs. The forecasting fields are saved at every 6 Hs. The perturbed model was defined adding $10 \%$ in the initial of all variables from control state. The difference between the averages calculated in entire temperature domain of the two models was chosen as a norm, and it was observed a random variation of $O\left(10^{-3}\right)$ on the full time integration interval.

Figure 2 and 3 show bred vectors at 06 UTC for the initial day (01/Jan/1982) and the last day (07/Jan/1982) of simulation. Large changes in the temperature field at the mid-latitudes regions can be observed, especially in the north of Canada and Europe.

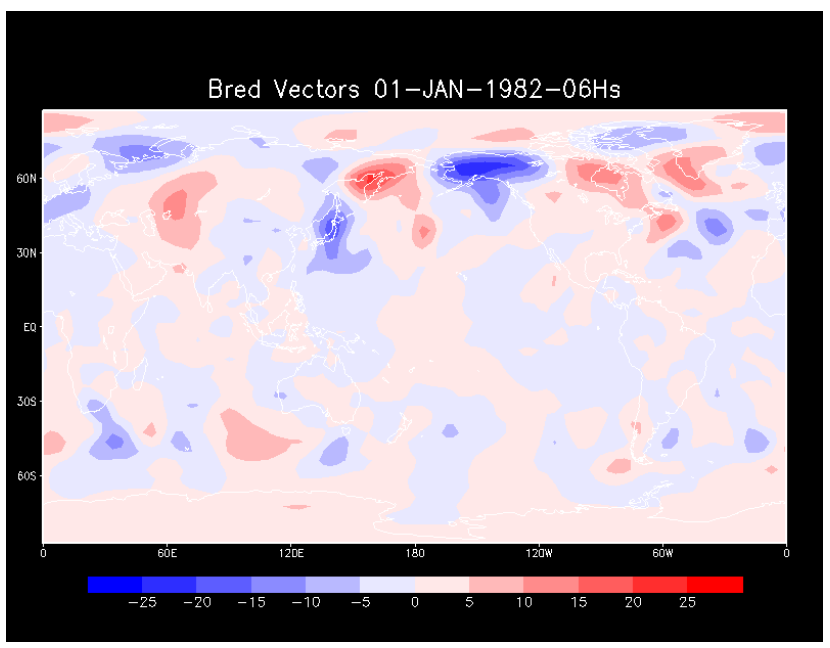

Figure 2 - Bred Vector: Temperature field at 01/Jan/1982, 06 UTC.

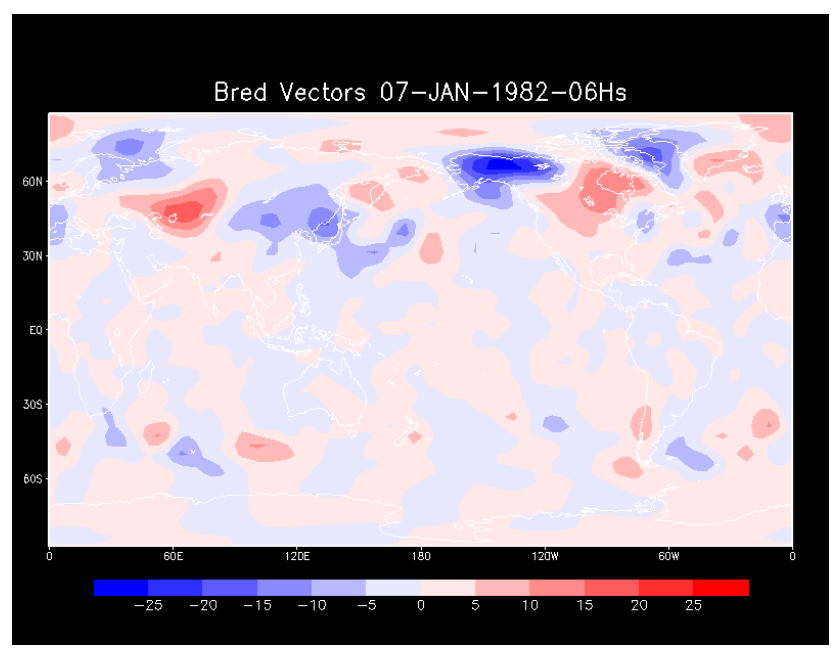

Figure 3 - Bred Vector: Temperature field 07/Jan/1982, 06 UTC. 
Figures 4 and 5 show the bred vectors for the vorticity at 06 UTC for the day (01/Jan/1982) and the last day (07/Jan/1982). Similar patterns can be visualized, but with smaller intensity for the both Hemispheres.

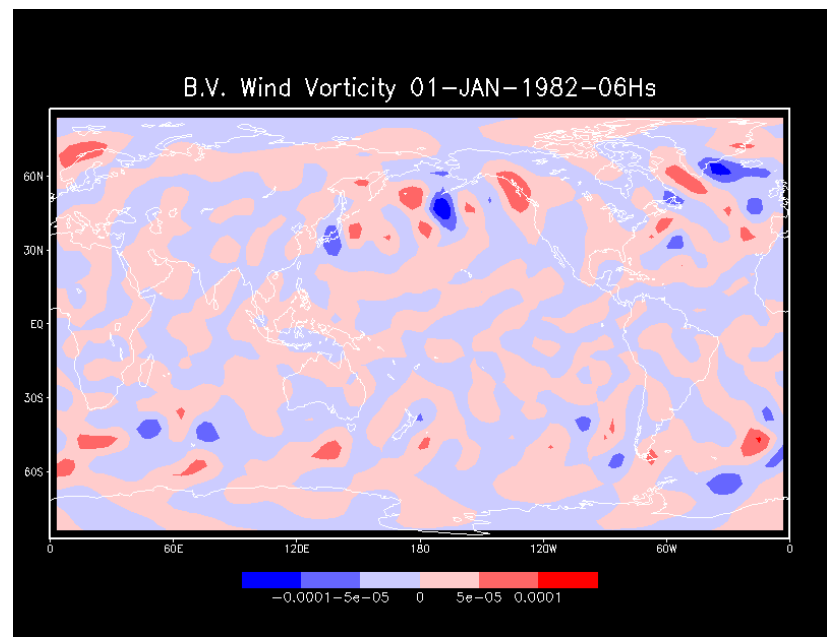

Figure 4 - Bred Vector: Vorticity field at 01/Jan/1982, 06 UTC.

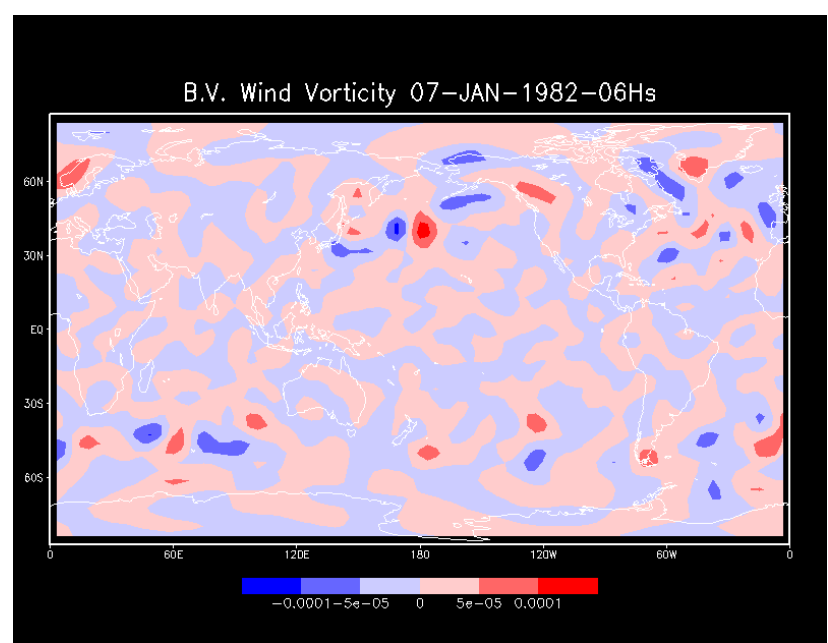

Figure 5 - Bred Vector: Vorticity field at 07/Jan/1982, 06 UTC.

Bred vectors computed by the surface pressure field is shown in Figures 7 and 6. The variation is more intense and the its significance is spread for entire globe. The differences become explicit the instabilities.

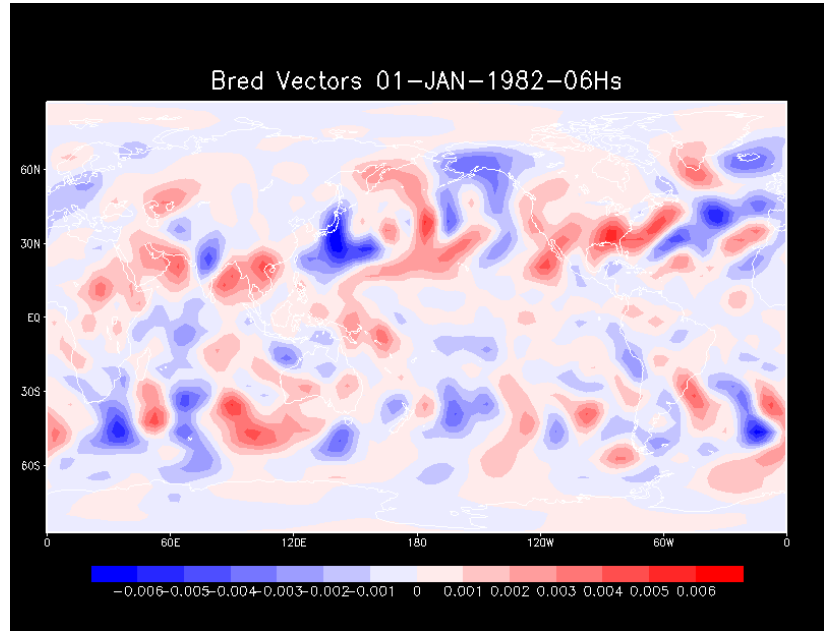

Figure 6 - Bred Vector: Pressure field at 01/Jan/1982, 06 UTC.

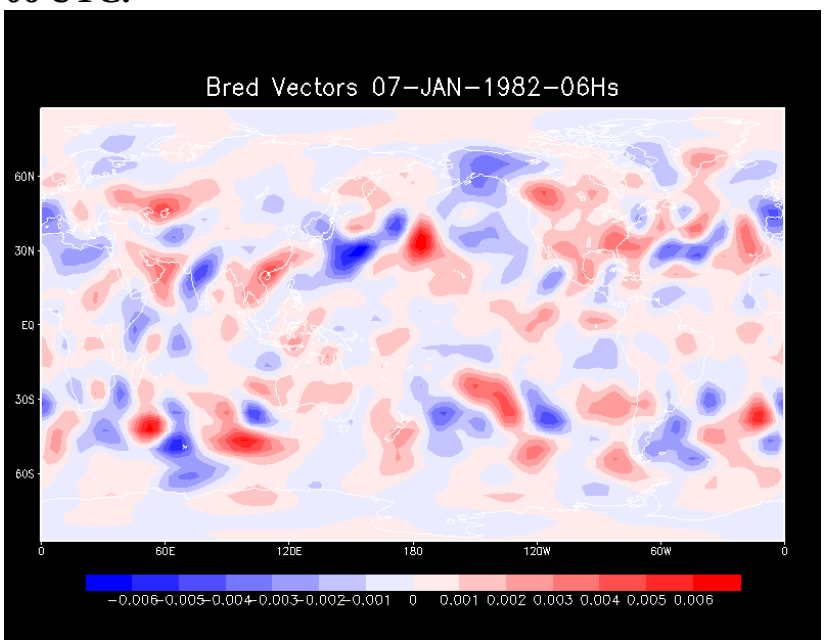

Figure 7 - Bred Vector: Pressure field at 07/Jan/1982, 06 UTC.

\section{CONCLUSION}

The breeding technique was applied to the SPEEDY model. The model simulates the atmospheric dynamics for the Earth. The bred vector method indicates the regions with high and low predictability, regions with small and large bred vector magnitude - respectively. Temperature and vorticity presented similar results. The analysis for the pressure field showed significant magnitudes for all planet.

\section{ACKNOWLEDGMENTS}

Authors would like to thanks CNPq: Brazilian agency for research support.

\section{REFERENCES}

[1] F. Molteni, and F. Kucharski, "Description of the ICTP AGCM (SPEEDY). Version 40," Abdus Salam International Centre for Theoretical Physics, Trieste, Italy. Consulted in March 2016. Available in http://users.ictp.it/ kucharsk/speedy_description/km_ 
ver40_appendixA.pdf

[2] R. S. Cintra, and H. F. de Campos Velho. "Global Data Assimilation using Artificial Neural Networks in Speedy Model". Proceedings of the 1st International Symposium on Uncertainty Quantification and Stochastic Modeling. pp 648-654, Maresias, São Sebastião, SP, Brazil, Febrary 2012.

[3] E. Kalnay, S. Yang, M. Peña, M. Cai, M. Hoffman. "Bred Vectors: A simplex tool to understand complex dynamics. Consulted in March 2016. Available in https://www.atmos.umd.edu/ ekalnay/pubs/Arakawa SymposiumKalnay.ppt.pdf

[4] Z. Toth, E. Kalnay. "Ensemble Forecasting at NCEP and the Breeding Method". Monthly Weather Review 1997 125:12, 3297-3319.

[5] M. Hoffman, E. Kalnay, J. Carton, S-C Yang. "Use of breeding to detect and explain instabilities in the global ocean". Geophys. Rese. Lett. 36, L12608. doi:10.1029/2009GL037729

[6] E. Kalnay, M. Corazza, M. Cai. "Are Bred Vectors the same as Lyapunov Vectors?". Symposium on Observations, Data Assimilation, and Probabilistic Prediction. Consulted in March, 2016. Available in https://ams.confex.com/ams/annual2002/techprogra m/paper_28833.htm 